\title{
Changing classroom culture, curricula, and instruction for proof and proving: How amenable to scaling up, practicable for curricular integration, and capable of producing long-lasting effects are current interventions?
}

\author{
Elena Nardi \\ University of East Anglia \\ Eric Knuth \\ University of Texas at Austin
}

Proof and proving continue to receive significant attention in the research literature (e.g., Stylianides, A., Bieda, \& Morselli, 2016; Stylianides G., Stylianides, A., \& Weber, 2017) as well as in educational reform initiatives and national standards documents (e.g., Common Core State Standards for Mathematics, 2010 in the USA; new GSCE and A level curricula in the UK, Department for Education, 2014, with their renewed emphasis on reasoning, particularly justification). Yet, despite the increased weight being placed on proof and proving, many students, of all ages, continue to struggle learning to prove (cf. Stylianides G. et al., 2017), and teachers as well struggle to facilitate their students' learning to prove (e.g., Bieda, 2010; Stylianides, G., Stylianides, A., \& Shilling-Traina, 2013). The work represented by the seven papers in this special issue, however, shows potential to enhance student learning in an area of mathematics that is not only notoriously difficult for students to learn and for teachers to teach, but also critically important to knowing and doing mathematics. 
Although the seven papers, and the intervention studies they report, vary in many ways-student population, content domain, goals and duration of the intervention, and theoretical perspectives, to name a few-they all provide valuable insight into ways in which classroom experiences might be designed to positively influence students' learning to prove. In our commentary, we highlight the contributions and promise of the interventions in terms of whether and how they present capacity to change the classroom culture, the curriculum, or instruction. In doing so, we distinguish between works that aim to enhance students' preparedness for, and competence in, proof and proving and works that explicitly foster appreciation for the need and importance of proof and proving. .Finally, we also discuss briefly the interventions along three dimensions: how amenable to scaling up, how practicable for curricular integration, and how capable of producing long-lasting effects these interventions are. We aim that our observations indicate productive (and needed) directions that continued efforts might take, particularly with regard to changes in classroom culture, curricula and instruction.

\section{Changing Classroom Culture, Curricula, and Instruction}

Systemic change with regard to the teaching and learning of proof and proving requires a multi-faceted effort, including considerations of teacher preparation and professional development, intentionally designed curricular materials (with respect to proof and proving), and instructional practices designed to meaningfully engage students in proving-related activities (e.g., conjecturing, exploring, justifying). In what follows, we briefly highlight aspects of each paper that fall under these considerations .

Changing Classroom Culture with respect to Proof and Proving 
Guala and Boero report on their work with future teachers, which is centered on the construct of Cultural Analysis of Content (CAC). CAC has two interrelated goals: to invite teachers to consider epistemological, historical, and anthropological aspects of mathematical content; and to embed an analysis of how said considerations influence a teacher's developing professional profile. A CAC perspective, for example, invites teachers to consider how different proofs of the same theorem may be constructed with reference to different theories. To assist teachers in this process, Guala and Boero adapt Habermas' (1998) construct of rationality and convert it into a list of criteria (e.g., validity of inferences, problem solving strategies, choice of communication means) that is then presented to teachers as they engage in proving activities (such as "Find the greatest common divisor of the product of three consecutive numbers").

A real strength of the approach illustrated in the paper, and one way in which the paper is separated from the other papers in this special issue, is the end goal of changing the "culture" of proving in schools. The majority of papers in this special issue focus primarily on enhancing student preparedness for proof (e.g., proof comprehension, proof generation), and less on enhancing student appreciation for proof. Although the two are interrelated, we contend that high appreciation for the need and importance of proof has the capacity to generate valuable momentum for developing proving competencies. For example, Nardi (1996) found that incoming mathematics undergraduates contest the didactic contract (Brousseau, 1997) presented to them upon arrival at university - a contract which takes such appreciation as a given. She found that students were frequently at odds with their lecturers on whether, for instance, an argument based on a diagram is acceptable or a so-called self-evident property of a function needs to be shown formally 
through resorting to definitions or previously proven theorems. And, when university lecturers were asked about the origins of this challenge to the didactic contract on proof and proving on the part of the students, their foremost reference was school classroom culture (Nardi, 2008).

Orchestrating a focus on proof appreciation requires a change in classroom culture with respect to proof and proving, a change that must start with teachers. In a recent doctoral study supervised by the first author (Kanellos, 2014; Kanellos, Nardi, \& Biza, 2013), the teacher's clear-eyed priority setting in favor of introducing mathematical proof to students as a culturally important and immensely useful tool within and outside mathematics-coupled with systematic engagement with a carefully eclectic mix of proving tasks in Algebra and Geometry-was the only plausible explanation for why an otherwise typical, mixed ability class had shown strong evidence of a learning trajectory towards deductive proof schemes (Harel \& Sowder, 2007). By attending to the epistemological, historical, and anthropological aspects of mathematical content—and proof, in particularGuala and Boero provide valuable evidence regarding the impact that participation in the CAC intervention has on the nuance of teachers' professional knowledge of proofs and arguments. Given teachers' often limited views regarding proof in school mathematics (e.g., Knuth, 2002a, 2002b), broadening the scope of teachers' professional knowledge through CAC may, ultimately, help teachers to change the culture of proving in schools in ways that may positively enhance both student preparedness and student appreciation for proof. Innovative Curricular Approaches to Proof and Proving

Several of the papers address issues that are more curriculum-related in nature (e.g., content-based interventions, task-based interventions), and illustrate how particular 
curricular experiences can positively impact important aspects of what students learn about proof and proving. In Fan et al., the authors focus on an understudied as well as critical aspect of geometric proofs-the construction of auxiliary lines. Auxiliary lines are often seen as a bit of a black box in geometric proofs and helping students to see the rationale for drawing a particular auxiliary line is often necessary. This paper addresses exactly this need. The authors take a novel approach: transformational geometry is used as a means towards helping students imagine more easily which lines, and how, facilitate proof production. Take, for example, the statement "the base angles in an isosceles triangle are equal." Through folding (or reflection, in the language of transformational geometry), students can see that the crease line AM divides the triangle into two congruent triangles (Figure 1).

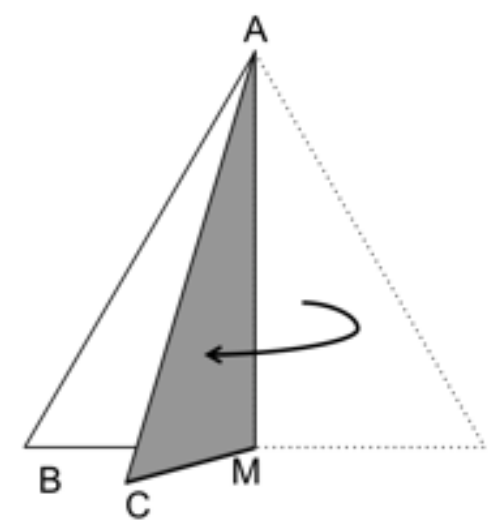

Figure 1. Snippet from Figure 1 in Fan et al., this Special Issue.

From this activity, students can see that by adding an auxiliary line (crease line AM, or line of reflection), two right-angled triangles, $\triangle \mathrm{ABM}$ and $\triangle \mathrm{ACM}$ become visible, which are congruent according to the hypotenuse-leg theorem $(\mathrm{AM}=\mathrm{AM}, \mathrm{AB}=\mathrm{AC}$, $\Varangle \mathrm{AMB}=90^{\circ}=\Varangle \mathrm{AMC}$ ). The congruency of the two triangles implies that the two base angles $\Varangle \mathrm{ABC}$ and $\Varangle \mathrm{ACB}$ are equal. Introducing a geometric transformation (in this case: reflection) 
can help students identify which auxiliary line, and why, may support the construction of a proof. The results of their intervention were mixed: no statistically significant differences were detected between the control and the experimental classes, even though the latter outperformed the former in $70 \%$ of the proving tasks. Fan et al. do suggest though that the potential of such an approach, especially if applied over a longer period of time so that its impact on the students' approaches to proof construction can be more solid, is worth further investigation.

Fiallo and Gutiérrez present results from an intervention designed to help students learn proof while studying trigonometry in a dynamic geometry software (DGS) environment. Initially we thought the authors' use of multiple theoretical perspectives (i.e., Boero et al.'s construct of cognitive unity of theorems, Pedemonte's structural and referential analysis of conjectures and proofs, Balacheff and Margolinas’ cKథ model, and Toulmin's argumentation scheme) was excessive, "theory overkill." In the end, however, we were convinced that the authors' use of the theories paid off. The result is an elaborate, if a little overly specified, examination of students' proof productions as they engage with carefully orchestrated activities in a DGS environment, and their overall progress from naïve empiricism to deductive proof. Additionally, the authors provide an informative set of four different cases of cognitive unity/rupture, corresponding to different ways of solving conjecture-and-proof problems: empirical cognitive unity ("Using examples does not favor the structural rupture necessary to move from perceptual argumentations about conjectures to deductive proofs", p. 21 of Online First version); referential rupture and empirical structural unity (despite difficulties in constructing a deductive proof evidence of generic example proof production emerges); referential unity and structural rupture 
("repeating previously used claims or statements, that lead to the construction of chains of statements that look like deductive proofs, but are incorrect", ibid.); and deductive cognitive unity ('Students' empirical actions are intended to check the accuracy of the conjectures, but argumentations and proofs are based on abstract general properties", ibid.).

In school mathematics, empirical-based reasoning often poses an obstacle to learning to prove, an obstacle that instruction must help students overcome (e.g., Sowder \& Harel, 1998; Stylianides, G., \& Stylianides, A., 2009; Zaslavsky, Nickerson, Stylianides, Kidron, \& Winicki, 2012). At the same time, an emerging body of research suggests that students who are able to strategically think about—and productively use-examples as they engage in proving-related activities may be better positioned to learn to successfully develop and evaluate mathematical arguments (e.g., Knuth, Zaslavsky, \& Ellis, accepted; Ozgur et al., accepted). Komatsu's intervention adopts this perspective in that he argues for the importance of student engagement in empirical examination as they investigate the validity of purported proofs and the truth of propositions. Komatsu's study demonstrates how students' empirical examinations of particular proof tasks (those with diagrams), and with particular teacher actions (e.g., prompting students to examine or draw diagrams different from the diagrams given in the task) can lead them to reject invalid proofs, to refute false propositions, and to modify proof/proposition conditions. Moreover, students' empirical examinations also served the purpose of engaging in authentic mathematical practices such as "discovery of cases that reject proofs and cases that refute statements, modification of the proofs, disclosure of hidden conditions to properly restrict the domains of the statements, and invention of more general statements that are true even for the refutation of the original statements" (p. 15 of Online First version). 


\section{Instructional Practices Designed to Enhance Proof Learning}

Several papers attend to various aspects of instructional practice, reporting interventions with foci that range from specific teacher actions to proof presentations. Mata-Pereira and da Ponte view generalizing and justifying as central reasoning processes, and the intervention they designed focuses on teacher actions-inviting, informing/suggesting, supporting/guiding, and challenging_as facilitators of these reasoning processes in the classroom. Although we wonder whether the instructional and task design principles they suggest would be applicable to any lesson preparation, not only to lessons predominantly focusing on proof and proving, the paper does offer evidence of the positive effect that endorsing these principles may generate. Their evidence focuses largely on reinforcing students' capacity to generalize and justify in the context of a sequence on linear equations. Their thoughtful design considers teacher aims (promoting a diversity of approaches and offering activities with different levels of challenge) as well as teacher actions (monitoring student progress, inviting explanations, probing for acceptable approaches to validation, encouraging the sharing of ideas, debating alternative and not necessarily instantly correct suggestions, offering support where needed for the task to progress and challenging with more demanding explorations where appropriate).

Gabel and Dreyfus set out from noting that research on different aspects of proof teaching at the university level, especially in relation to how mathematics lecturers present proofs to their students, is mostly descriptive in nature, and their aim is to evaluate what makes a proof presentation clear and effective, and subsequently, what intervention may be needed to have the desired effect. They present a case study from a university number theory course in which they adopted Perelman's New Rhetoric (PNR, Perelman \& 
Olbrechts-Tyteca, 1969) as a theoretical framework for analyzing the "flow" of a proof. "Flow of a proof" is defined as the outcome of the choices made by a lecturer with regard to: presentation of the logical structure of a proof; how informal features of a proof such as examples, intuitions, and diagrams are incorporated in the presentation of a proof; and mathematical and pedagogical contextual factors. They propose analyzing the flow of a proof both from a global perspective (chunking a proof into individual modules) and from a local perspective (examining singular arguments in the proof, formal or informal).

Specifically, they use two features of PNR (scope, i.e. how a lecturer may regulate the density in the presentation of a proof according to the audience that is being addressed; and presence, i.e. what degree of emphasis, frequency of repetition etc. a lecturer may choose in the presentation of a proof) to analyze the flow of a proof and change it. They observe a lecturer during a Number Theory lecture that centered on the proof of the theorem, "The greatest common divisor (gcd) of two integers $a, b$, at least one of which is not 0 , equals the smallest natural number of the form $m a+n b$, where $m, n$ are integers: $\operatorname{gcd}(a, b)=\min \{m a+n b>0: m, n \in \mathrm{Z}\}$," and, after interaction following the first delivery of the lecture, they demonstrate how the lecturer was able to change the flow of a presented proof both in terms of scope and presence. The intervention focused on suggestions on how to improve the flow of the proof through smoother transitions between the proof modules and more emphasis on what necessitated the use of the remainder theorem, to name a few. Observed changes in the flow of the lecture concerned both scope (e.g., the non-formal argumentation leading to the proof in the post-intervention lecture caused "a noticeable change of scope and organization changing the global flow of the proof substantially"; p. 14 of Online First version) and presence (e.g., there was evidence of the lecturer's raised 
"awareness of the fact that the distinction between existence and constructive proof is not trivial for the students" and this led to endowing "this proof element with a strong degree of presence"; p. 16).

While the paper does not directly address whether the intended learning outcomes were achieved in relation to student comprehension and affective response to proof and proving tasks, we found the authors' proposed use of PNR compelling. We also found the comparison of PNR to other constructs used for similar purposes in our field informative, and it served to highlight the potential strengths of PNR for teaching proof.

Finally, Roy, Inglis, and Alcock present results from a study that serves as a cautionary tale about instructional interventions. In particular, they report results from an intervention in which multimedia resources were designed with the intention to help undergraduate students comprehend proofs. The multimedia resource that the study focused on, "e-proofs," is a resource which captures the explanations that a lecturer "would ordinarily offer verbally", but "allows students to engage more fully with these by making them replayable and by highlighting the parts of the proof to which they referred" (p. 4 of Online First version). An e-proof offers multiple explanation screens, with each screen focusing attention using "greying out, boxes and arrows" and being accompanied "by a short audio commentary that could be played by clicking a button, and replayed as many times as desired" (ibid.).

On the surface, it seemed reasonable, and expected, that the e-proofs would improve students' proof comprehension. Yet, unexpectedly, students actually exhibited poorer performance than those students who engaged with the same proofs presented without the resources. The authors - through drawing on extensive datasets collected through eye- 
tracking technology—conclude that the extra supporting resources may disrupt the way in which students process and organize the information presented in the proofs, and as a result, may limit their proof comprehension. Although not the intention at the outset, the authors also raise important questions about the responsibilities of instructors to evaluate the effectiveness of instructional resources made available to students. This is especially the case given the growing abundance of easily accessible resources (e.g., web-based curricular materials, on-line videos). The paper also serves as a poignant cautionary tale: evaluating the effectiveness of instructional resources needs to be done in far more robust and systematic ways than merely relying on students' self-reported learning outcomes and satisfaction with the resources.

\section{Enhancing Students' Preparedness and Appreciation for Proof and Proving}

The various papers demonstrate, with varying degrees of success, interventions that target either students' preparedness for proof and proving or teachers' instructional practices for teaching proof and proving. In the first type, the interventions depended upon the use of specific tasks designed to evoke particular student actions (e.g., construction of auxiliary lines, refutation of purported proofs) or of specific curricular environments (e.g., dynamic geometry software, transformational geometry). In the second type of intervention, approaches focused on ways to enhance the presentation (and,

ultimately, student comprehension) of proofs, teachers' actions related to engaging students in proof and proving, or teachers' appreciation for proofs and proving. In both types of intervention, we see examples of students who are seemingly better prepared for proof and proving as a result. Although the goal of these studies, understandably, was not to produce broadly generalizable interventions, nevertheless, we did wonder whether 
these works could claim longer-term, deeper, and broader impact on students' preparedness for proof and proving in typical school contexts. Also, in both types, a focus on fostering appreciation for proof and proving was largely missing-with the exception as we mentioned earlier of the Guala and Boero paper.

\section{Concluding Remarks}

As a collective, the papers in this special issue provide a picture of promising approaches to classroom-based interventions that have the potential to enhance the teaching and learning of proof and proving. Gabel and Dreyfus noted (based on a comment from Fukawa-Connelly, Johnson, \& Keller, 2016) that "new policies and reforms in mathematics education will not gain traction unless they are perceived as practical and feasible in the eyes of the mathematicians [and school mathematics teachers] delivering

the instruction" (p. 17 of Online First version). As we consider the interventions reported in this special issue, the aforementioned comment underscores the necessity for interventions that are amenable to scaling up, practicable for incorporation into existing curricular structures, and capable of producing long-lasting, positive effects (Stylianides, A. \& Stylianides, G., 2013).

There is a long history of research that has documented the challenges of teaching and learning proof, and, although such research has provided invaluable insight into these challenges, students continue to struggle with learning to prove, curricula continue to provide inadequate opportunities for engaging students with proof, and teachers continue to struggle with teaching proof. As Stylianides et al. (2017) note, "research thus far has offered inadequate support to teachers, teacher educators, and curriculum developers (including textbook authors) about how they might address problems of practice in the 
area of proof" (p. 252). The papers in this special issue, and classroom-based intervention research with respect to proof and proving, show promise to make further progress into the perennial concern in mathematics education that students fail to understand the nature of evidence and justification in mathematics. The special issue papers mark an exciting, and relatively new, direction in research on proof and proving, a direction that we think will provide the foundation and guidance needed for making proof and proving a consistent and regular part of the mathematics education of all students.

\section{References}

Balacheff, N., \& Margolinas, C. (2005). cKф modèle de connaissances pour le calcul de situations didactiques. In A. Mercier, \& C. Margolinas (Eds.), Balises pour la didactique des mathématiques (pp. 75-106). Grenoble, France: La Pensée Sauvage.

Bieda, K. (2010). Enacting proof-related tasks in middle school mathematics: Challenges and opportunities. Journal for Research in Mathematics Education, 41, 351-382.

Boero, P., Garuti, R., Lemut, E., \& Mariotti, M. A. (1996). Challenging the traditional school approach to theorems: A hypothesis about the cognitive unity of theorems. In L. Puig, \& A. Gutiérrez (Eds.), Proceedings of the 20th PME Conference (vol. 2, pp. 113-120). Valencia, Spain: PME.

Brousseau, G. (1997). Theory of didactical situations in mathematics. Dordrecht, NL: Kluwer.

Council of Chief State School Officers. (2010). Common Core State Standards for Mathematics. Washington, DC: Council of Chief State School Officers.

Department for Education (2014). Mathematics programmes of study: key stages 1 and 2: National curriculum in England: 
https://www.gov.uk/government/publications/national-curriculum-in-englandmathematics-programmes-of-study

Fukawa-Connelly, T., Johnson, E., \& Keller, R. (2016). Can math education research improve the teaching of abstract algebra? Notices of the American Mathematics Society, 63, 276-281.

Harel, G., \& Sowder, L. (2007). Toward comprehensive perspectives on the learning and teaching of proof. In F. Lester (Ed.), Second handbook of research on mathematics teaching and learning (pp. 805-842). Charlotte: Information Age Publishing.

Kanellos, I. (2014). Secondary students' proof schemes during the first encounters with formal mathematical reasoning: Appreciation, fluency and readiness. Unpublished doctoral thesis: University of East Anglia, UK.

Kanellos, I., Nardi, E., \& Biza, I. (2013). The interplay between fluency and appreciation in secondary students' first encounter with proof. In A.M. Lindmeier \& A. Heinze (Eds.), Proceedings of the 37th Conference of the International Group for the Psychology of Mathematics Education (PME) (Vol. 5, pp. 84). Kiel, Germany: PME.

Knuth, E. (2002a). Secondary school mathematics teachers' conceptions of proof. Journal for Research in Mathematics Education, 33(5), 379-405.

Knuth, E. (2002b). Teachers' conceptions of proof in the context of secondary school mathematics. Journal of Mathematics Teacher Education, 5(1), 61-88.

Knuth, E., Zaslavsky, O., \& Ellis, A. (Accepted). The role and use of examples in proving-related activities. To appear in Journal of Mathematical Behavior.

Nardi, E. (1996). The novice mathematician's encounter with mathematical abstraction: Tensions in concept image construction and formalisation Unpublished doctoral 
thesis, University of Oxford, UK (available at http://www.uea.ac.uk/ m011 and https://ora.ox.ac.uk/objects/uuid:19d55975-7af9-4ed4-ab98-3be18da31e16).

Nardi, E. (2008). Amongst mathematicians: Teaching and learning mathematics at university level. New York: Springer.

Ozgur, Z., Vinsonhaler, R., Dogan, M., Knuth, E., \& Ellis, A. (Accepted). From examples to proof: Purposes, strategies, and affordances of example use. To appear in Journal of Mathematical Behavior.

Pedemonte, B. (2005). Quelques outils pour l’analyse cognitive du rapport entre argumentation et démonstration. Recherches en Didactique des Mathématiques, 25(3), 313348.

Perelman, C., \& Olbrechts-Tyteca, L. (1969). The new rhetoric: A treatise on argumentation (J. Wilkinson \& P. Weaver, Trans.). Notre Dame, IN: University of Notre Dame.

Sowder, L., \& Harel, G. (1998). Types of students' justifications. Mathematics Teacher, 91, 670-675.

Stylianides, A. J., Bieda, K. N., \& Morselli, F. (2016). Proof and argumentation in mathematics education research. In A. Gutiérrez, G. C. Leder, \& P. Boero (Eds.), The Second Handbook of Research on the Psychology of Mathematics Education (pp. 315-351).

Rotterdam, The Netherlands: Sense Publishers.

Stylianides, A. J., \& Stylianides, G. J. (2013). Seeking research-grounded solutions to problems of practice: Classroom-based interventions in mathematics education. ZDM, 45, 333-341. 
Stylianides, G. J., \& Stylianides, A. J. (2009). Facilitating the transition from empirical arguments to proof. Journal for Research in Mathematics Education, 40, 314-352.

Stylianides, G. J., Stylianides, A. J., \& Shilling-Traina, L. N. (2013). Prospective teachers' challenges in teaching reasoning-and-proving. International Journal of Science and Mathematics Education, 11, 1463-1490.

Stylianides, G. J., Stylianides, A. J., \& Weber, K. (2017). Research on the teaching and learning of proof: Taking stock and moving forward. In J. Cai (Ed.), Compendium for Research in Mathematics Education (pp. 237-266). Reston, VA: National Council of Teachers of Mathematics.

Toulmin, S. E. (2003). The uses of argument (updated edition of the 1958 book). Cambridge, UK: Cambridge University Press.

Zaslavsky, O., Nickerson, S., Stylianides, A., Kidron, I., \& Winicki, G. (2012). The need for proof and proving: Mathematical and pedagogical perspectives. In G. Hanna \& M. de Villiers (Eds.), Proof and proving in mathematics education (pp. 215-229). New York, NY: Springer. 